\title{
Use of Wireless Sensor and Microcontroller to Develop Water-level Monitoring System
}

\author{
Abdullah-Al-Mamun ${ }^{1}$, Nasim Ahmed ${ }^{2}$, Nizam Uddin Ahamed ${ }^{3 *}$, \\ S. A. M. Matiur Rahman ${ }^{4}$, Badlishah Ahmad $^{2}$ and Kenneth Sundaraj ${ }^{5}$ \\ 'Electronics Division, Atomic Energy Centre, Dhaka-1000, Bangladesh \\ ${ }^{2}$ School of Computer and Communication Engineering, Universiti Malaysia Perlis, Malaysia \\ ${ }^{3}$ Faculty of Manufacturing Engineering, Universiti Malaysia Pahang, 26600 UMP Pekan, \\ Pahang, Malaysia; ahamed1557@hotmail.com \\ ${ }^{4}$ College of Computer Science and Information System, Najran Univeristy, Kingdom of Saudi Arabia \\ ${ }^{5}$ Al-Rehab Research Group, Universiti Malaysia Perlis, Malaysia
}

\begin{abstract}
This paper presents the design and development process of Wireless Data Acquisition System (WiDAS) which is a multisensor system for water level monitoring. It also consists of a microcontroller (ATMega8L), a data display device and an ultrasonic distance sensor (Parallax Ping). This wireless based acquisition system can communicate through RF module ( $T x-R x)$ from the measurement sources, such as sensors and devices as digital or analog values over a period of time. The developed system has the option to store the data in the computer memory. It was tested in real time and showed continuous and correct data. The developed system is consisting of a number of features, such as low energy consumption, easy to operate and well-built invulnerability, which cangive successful results to measure the water level. Finally, its flexibility facilitates an extensive application span for self-directed data collection with trustworthy transmission in few sparse points over huge areas.
\end{abstract}

Keywords: Microcontroller, Water-level Monitoring System, WiDAS, Wireless Sensor

\section{Introduction}

To monitor the level of water is often a key requisite in swimming pool, dam, river, coastal, fisheries, irrigation and canal control, oceanography and so on. Moreover, to detect the water level of water reservoir through wire communication is not a flexible and tedious ways. For this purpose a robust, real time, portable and easy to operating system is needed for monitoring the level of water. There are much of the technologies used today to execute the basic tasks as water-level measurement, water-quality characterization and so on. For monitoring the level of water, the system is a kind of structure which measures water deepness through ultrasonic sensor technology ${ }^{1}$. Modern microcontroller and wireless sensor can provide a range of solutions for the automated monitoring of water levels in many applications ${ }^{10}$. In most of the cases, costly radio modems are employed for fastest access of remote data, because it provides a long-distance and reliable radio link between the sensor networks. Simultaneously, the processed digitized data is transmitted via a wireless network (while using wireless system) to a remote location or device. In another sense, the transmission step is made over a wireless network control channel using GSM, CDMA, 3G, Wi-Fi, RF modules or Zigbee digital technology $y^{3,6,14}$. Also, a WiDAS (Wireless Data Acquisition System) which is a remote-sensing device designed to obtain multi-angle infrared remote-sensing data in remote distance ${ }^{2}$.

Till now, several attempts have been made to provide an effective monitoring system to observe the level of water. A number of papers reported with different technologies to develop this kind of monitoring system.

${ }^{*}$ Author for correspondence 
Like, Maqbool et al. ${ }^{9}$ developed wireless system using Zigbee, 74HC14 Inverter and an IC (74HC14 hex inverting Schmitt trigger) $)^{9}$. Then, Greswell et al. ${ }^{4}$ developed a simple, inexpensive, portable and wired water level monitoring system for multiple applications ${ }^{4}$. In one paper, author constructed a water level measuring system by the sense of capacitance of eleven terraces ${ }^{13}$. Moreno et al. ${ }^{11}$ presented the design specification of an active water level monitoring system using 8051 microcontroller and Digital Input/Output (DIO-1) board11. Yan et al. ${ }^{15}$ developed an intelligent coal-mine water level measuring and monitoring system using the Wireless Sensor Networks (WSNs), and microcontroller PIC. There are several functions in their system, for example measurement, display unit, alarm and communication channels15. Then Yi-Bing designed a long-range monitoring system of water level using RS232 and GPRS network to the data terminals ${ }^{16}$. Another GPRS wireless module based system developed by Yang where he used G20 GPRS module, and integrating AVR Atmegal 6 control chip ${ }^{17}$. Rosolem et al. ${ }^{12}$ described a low cost Fiber Optic Bending optical sensor based water level monitoring systems which can measure up to $10 \mathrm{~m}$ or more and choose the appropriate membrane ${ }^{12}$. In addition, some other research works has done with these related and common techniques to develop a robust system for monitoring water level ${ }^{5,7,8,18}$.

In very few studies, however, have researchers attempted to design the water level monitoring system using following techniques and devices: a complete wireless data acquisition system made up with sensors, analog frontend, a MCU, wireless transceivers, and a remote host, microcontroller ATMega8L, RF module (Tx-Rx), LCD display, ultrasonic distance sensor (parallax ping), water container with nozzle and buzzer (for alarm). The entire design plan of the current system assembles on these models and also takes advantage of the comparatively recent accessibility of miniature, stand-alone, easy-to-use and low-price monitoring system that allow the entire water level measuring system to be diminished in size and become independent of a centralized logging apparatus. Even though it is feasible for those adequately skilled and equipped to obtain the design one step further to include the various electronics equipments, those are described by the previous researchers, and we believe our approach is appropriate for the broad audiences since it needs less electronic assembly expertise. Finally, in order to search for an improved solution to the water level measuring problem, this paper proposes the design, construction, implementation and validation of an easy and inexpensive sensing system that may be readily adjusted to suit a large range of water level monitoring applications.

The remainder of the paper is organized as follows; section 2 presents an explanation of the methodology used to develop and evaluate the system. Section 3 depicts the results from the developed system and the section 4 discusses the overall outcomes from the new findings of the research. Finally, section 5 provides concluding remarks and future research directions.

\section{Methodology}

A number of software and hardware implementation techniques were used to design and develop the system. A microcontroller, an ultrasonic distance measurement sensor and a pair of RF module have been used to design the system. Ultra sonic sensor used to detect the water level, then the data will go to transmit and receive through the RF module and the whole procedure is going control by the microcontroller. Figure 1 shows the block diagram of the system.

Ultrasonic sensor generates high frequency sound waves and evaluates the echo from the water level of the

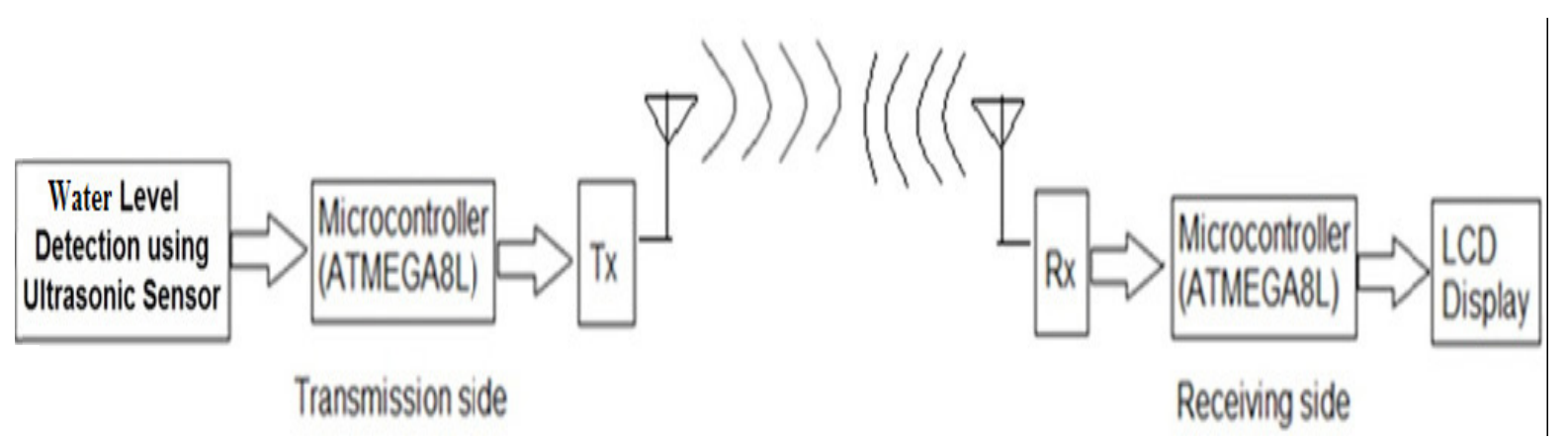

Figure Block diagram of the designed system. 
reservoir which is received back by the sensor. Ultrasonic sensor has the following specifications, Supply voltage: 5 VDC (Volts Direct Current), supply current: $30 \mathrm{~mA}$ type (35 $\mathrm{mA} \max$ ), range: $2 \mathrm{~cm}$ to $3 \mathrm{~m}$, input trigger: positive Transistor-Transistor Logic (TTL) pulse, $2 \mathrm{mS} \min (5 \mu \mathrm{S}$ type), echo pulse: positive TTL pulse (115 $\mu \mathrm{S}$ to $18.5 \mathrm{mS})$, echo hold-off: $750 \mu$ s from fall of trigger pulse, burst frequency: $40 \mathrm{kHz}$ for $200 \mu \mathrm{S}$ and size: $22 \mathrm{~mm} \mathrm{H} \mathrm{x} 46 \mathrm{~mm}$ $\mathrm{W} \times 16 \mathrm{~mm}$ D. Figure 2 represents how the ultrasonic sensor working within the aquiition system. Sensors Module calculates the time interval between sending the signal and receiving the echo to determine the distance of water level height from the bottom of reservoir. At the transmission side, a microcontroller module has been attached to transmit the sensor data through wireless TX module. The schematic of the microcontroller module with transmission and receiving sides are shown in Figure 3 (a) and Figure 3 (b). In this system the low-power 8-bits CMOS microcontroller (ATMega8L) have attached (based on the AVR RISC architecture) which is manufactured by ATMEL, San Jose, CA.

At the receiving side, another microcontroller module has been also used to receive the sensor data. An integrated Liquid Crystal Display (LCD) associated with microcontroller module is also used for real time display of water level data acquired from sensor. The Light Emitting Diode (LED) is used to indicate the different level of water and a buzzer (beeper) will be active in the case of overflow and empty level. The flow diagram of multi-sensor data processing is shown in Figure 4.

Software design includes developing algorithm for the system, allocating memory blocks as per functionality, writing the separate routines for different interfacing devices such as UART, LCD, UT sensor and testing them on the designed hardware. The control program is written in BASIC programming language on BASCOM-AVR (freeware program). The overall Instrument examines outcome states by using of this microcontroller BASCOMAVR language program.

\section{Results}

The aim of this field-test is to provide a long-term monitoring system to achieve continuous and correct information about water level. To fulfill this objective, the following test was conducted to evaluate the success of the developed monitoring system. The system connected with sensor proposed has a particular design to be simple, trustworthy, and low-cost which is fit to be used in swimming pool, embankment dams, river, coastal tanks, and

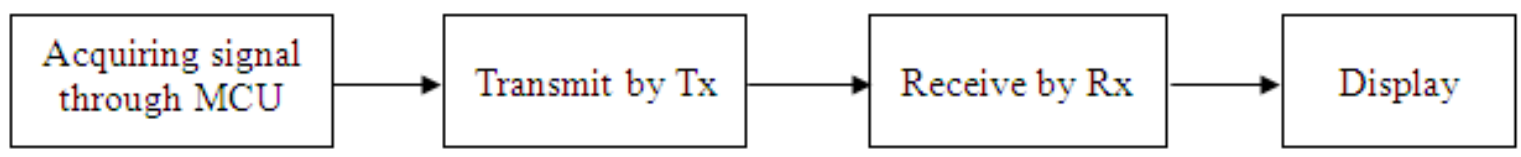

Figure 2. Block diagram showing the ultrasonic sensor unit connected with the data Acquisition system.

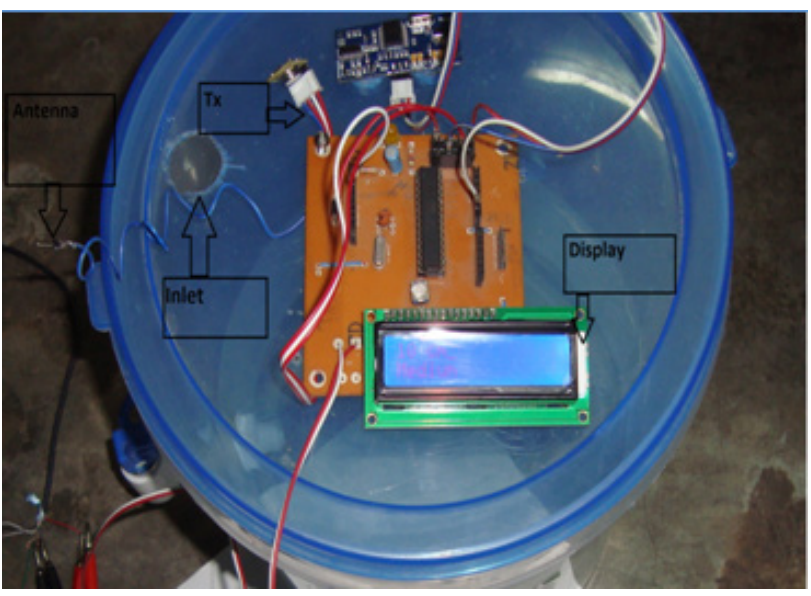

(a)

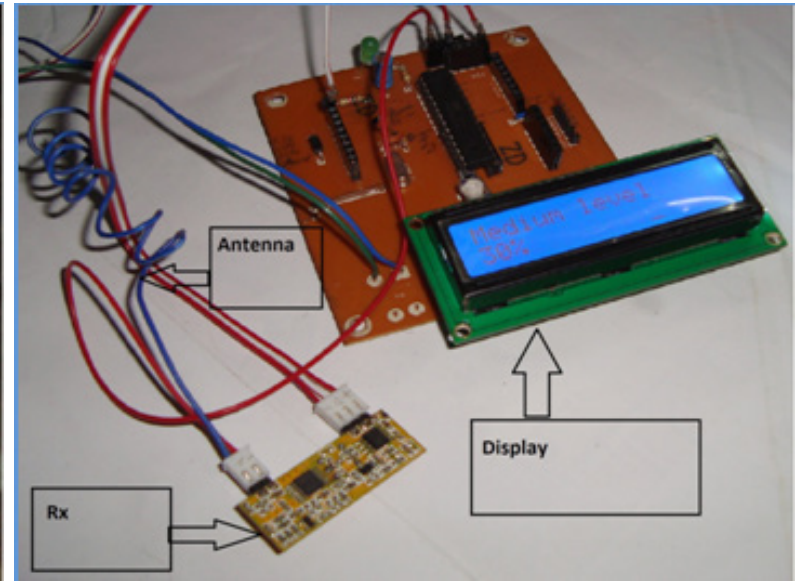

(b)

Figure 3. Developed system (a) Transmission side (b) Receiving side. 


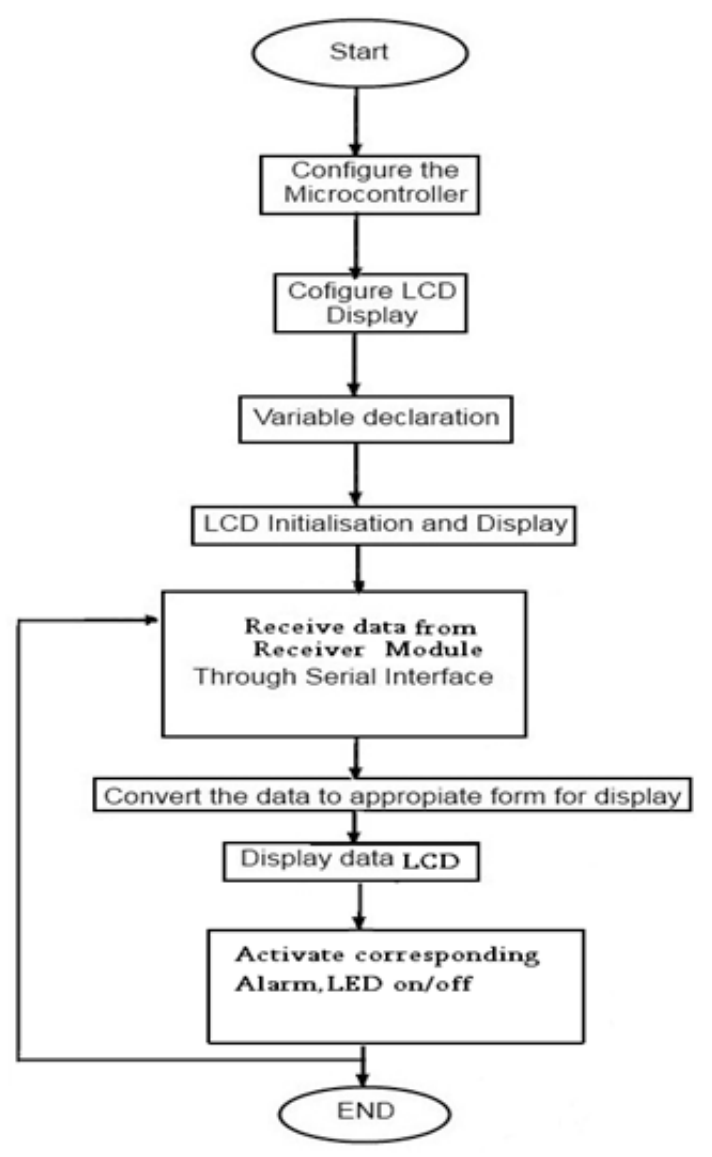

(a)

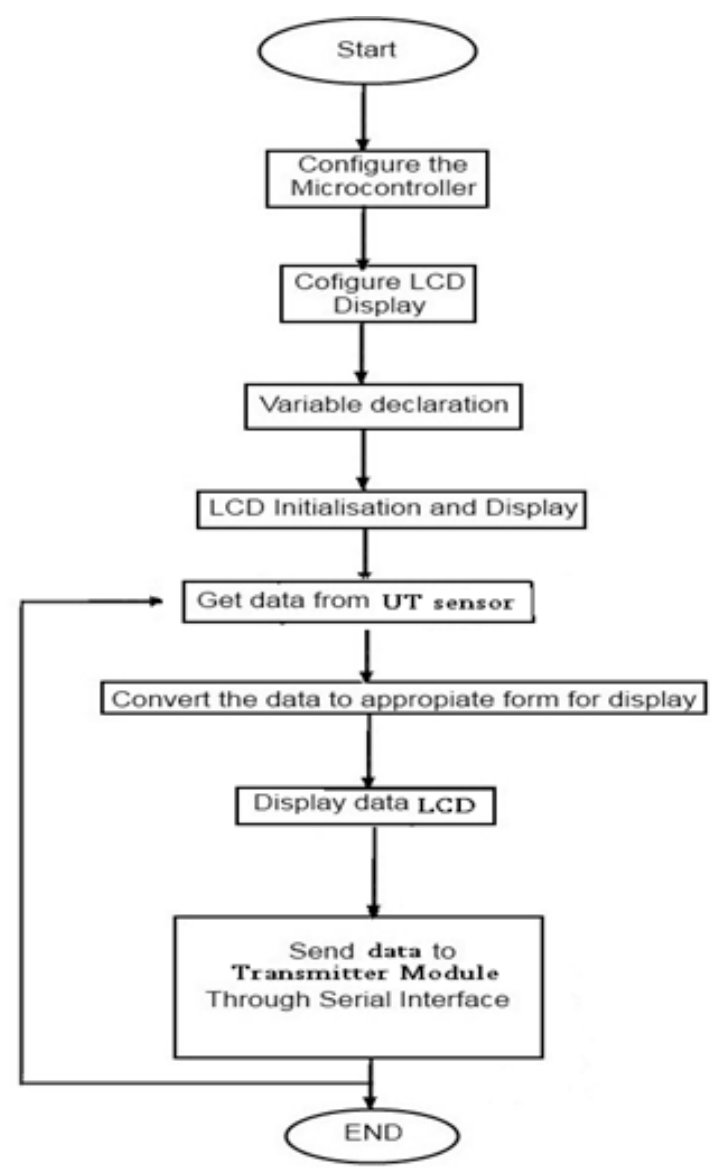

(b)

Figure 4. Flow diagram (a) Transmitter Module (b) Receiver Module.

reservoirs. This section presents the implementation and experimental results performed by field tests.

The entire instruments were placed in the exact location for the detection of water level and all the electrical connections within the system were checked. A 23-centimeter lengthen bucket was filled up with the water which was treated as a conventional water tank. After that the level of water was measure by using the ultrasonic distance sensor and calculated the distance in centimeter-scale. In addition, the reading of water level in the transmitting side shown in centimeter. On the other hand at the receiving end the reading of water level was calculated by the corresponding percentage.

The main function of the system is to sense the level of the water where the level was expressed in five different levels: empty, low, medium, high and full (Table 1). The different levels of water were determined by the appropriate color of the LED. The indications were as follows: full, high and medium levels show the green signal; low and empty is red signal; and if water level is less than $50 \%$ is red signal, Also, the level was determined by the responded sound from buzzer as full, low and empty means it will give sound. Finally, the data acquired by the system was satisfactory and the reading was accurate which has compared by the previous experiments.

\section{Discussion}

The developed system has been practically verified as a strong and reliable instrument for measuring water level in a wide range of applications. It has fulfilled the objective which was success to transfer data at the short-range using wireless communication network, is low-cost and is flexible to use. Also, the embedded wireless receiver module of multipoint short-range wireless data collection and transmission network provides a low-powered and high-performance 
Table 1. Observation of the level of water with the developed system

\begin{tabular}{lcclll}
\hline Level of water (cm) & $\begin{array}{c}\text { Level detection } \\
\text { (displayed in } \\
\text { LCD 1) }\end{array}$ & Level of water (\%) & $\begin{array}{c}\text { Level detection } \\
\text { (displayed in } \\
\text { LCD 2) }\end{array}$ & Lights color (LED) & Buzzer \\
\hline$<4 \mathrm{~cm}$ & $0-4 \mathrm{~cm}$ & $100 \%$ & Full & Green (double) & Yes \\
$<6 \mathrm{~cm}$ & $5-6 \mathrm{~cm}$ & $90 \%$ & High & Green & No \\
$<8 \mathrm{~cm}$ & $7-8 \mathrm{~cm}$ & $70 \%$ & Medium & Green & No \\
$<10 \mathrm{~cm}$ & $9-10 \mathrm{~cm}$ & $50 \%$ & Medium & Green & No \\
$<12 \mathrm{~cm}$ & $11-12 \mathrm{~cm}$ & $30 \%$ & Medium & Blue & No \\
$<14 \mathrm{~cm}$ & $13-14 \mathrm{~cm}$ & $10 \%$ & Low & Red & Yes \\
$<20 \mathrm{~cm}$ & $15-19 \mathrm{~cm}$ & $0 \%$ & Empty & Red (double) & Yes \\
\hline
\end{tabular}

wireless data communication system, which works and gathered data from sensor through RF modules. In order to meet all these requirements, a low-cost, versatile, and portable WiDAS has designed. In addition, a microcontroller based ultrasonic sensor system with $\mathrm{Tx}$-Rx has been developed for measuring water level. The reason for design the process is to receive data from ultrasonic sensors and to process by microcontroller, and the data can send through transmitter to another microcontroller. After receiving by a receiver data can display on a LCD display. The LED will indicate the different level of water and a buzzer will get active in the case of overflow and empty level.

In most of the techniques and applications discussed here, a related or proper commercial device is relatively rare available in the market and therefore the measurements we have carried out may only be accomplished using the following approaches:

1. The developed system has been achieved a shortrange of data transmission, and presented a technical approach for water level measuring decision,

2. The profiling of the software implementation was obtained using the BASCOM-AVR development environment,

3. The components of developed WiDAS systems include: i) sensors that convert physical parameters to electrical signals, ii) signal conditioning circuitry to convert sensor signals into a form that can be converted to digital values, and iii) analog-to-digital converters, which convert conditioned sensor signals to digital values.

4. Finally, this paper shows a real-time system modules which have been designed to permit low cost, synchronicity, expandability, customizability and give alarm.

\section{Conclusion and Future Works}

The current research work illustrates the design and performance of a real-time monitoring system for measuring water level. The developed system mainly highlights the functional characteristics with wireless network capacity, sensor hardware compatibility (ultrasonic sensor), lowcost production and efficient capture. The system also has the low-power consumption and has negligible impact on the environment. The tested result from the system reports good outcomes which is energy resourceful, has strong communication ability, and presents real-time measurement accuracy. The WiDAS can be further improved on the following aspects: combination of more sensors for large measuring area, further improved by utilizing the new generation of microcontrollers, to establish an intelligent power management system, to add temperature function will be added and to improve the overall performance of this system.

\section{References}

1. Arshad MR. Recent advancement in sensor technology for underwater applications. Indian J. Marine Sci. 2009; 38(3):67-273.

2. Fang L, Liu Q, Xiao Q, Liu Q, Liu Z. Design and implementation of airborne wide-angle infrared dual-mode line/ area array scanner in Heihe experiment. Advances in Earth Science. 2009; 24:696-705.

3. Glatz PM, Hörmann LB, Steger C, Weiss R. Designing perpetual energy harvesting systems explained with rivermote: A wireless sensor network platform for river monitoring. Electron J Struct Eng. 2010; Special Issue: Wireless Sensor Networks and Practical Applications:55-65.

4. Greswell R, Ellis P, Cuthbert M, White R, Durand V. The design and application of an inexpensive pressure monitoring 
system for shallow water level measurement, tensiometry and piezometry. J Hydrol. 2009; 373(3-4):416-25.

5. Hongkun Z, Yongrui Z. Design of water level monitoring system for rubber dam based on Zigbee. Journal of Suzhou University. 2010; Engineering Science Edition, 1: 017.

6. Huang X.-y, Li Z.-d, Meng D.-s. Application of GSM short message in communication of water-level remote-system. Journal of Waterway and Harbor. 2004; Z1:123-4.

7. LI Y.-y, LI J.-z. Wireless monitoring system design of temperature and water level of solar water heater with wireless sensor. Telecom Power Technology. 2012; 6:019.

8. Manuchehri H, Aminian E. Design and construction of a water level and temperature monitoring system for fish farms. Journal of Fisheries (Iranian Journal of Rresources). 2010; 63(3):219-27.

9. Maqbool S, Chandra N, Dagadi S. ERPWS: An energy efficient routing protocol for conductive sensor based water Level monitoring and control system using Zigbee and 74HC14 Inverter. Int J Mod Educ Comput Sci. 2013; 5(6):31.

10. McCulloch J, McCarthy P, Guru SM, Peng W, Hugo D, Terhorst A. Wireless Sensor Network Deployment for Water use Efficiency in Irrigation. Proceedings of the workshop on Real-world wireless sensor networks; 2008; ACM. p. 46-50.

11. Moreno M, Salazar A, Gijon R, Attarzadeh F, Mahajan M, Gupta A, Kesari P. Active Vehicle Water Level Monitoring
System, Proceedings of the ASEE Gulf-Southwest Annual Conference; 2008 Mar; The University of New MexicoAlbuquerque. p. 26-8.

12. Rosolem JB, Dini DC, Penze RS, Floridia C, Leonardi AA, Loichate MD, Durelli AS. Fiber Optic Bending Sensor for Water Level Monitoring: Development and Field Test: A Review. IEEE Sensor J. 2013;13(11):4113-20.

13. Shengqing C. Investigation on water level measuring system of laddered capacitor. Journal of Ningxia University. 2005; 4:009.

14. Sunkpho J, Ootamakorn C. Real-time flood monitoring and warning system. Songklanakarin Journal of Science \& Technology. 2011; 33(2):227-35.

15. Yan J-J, Liu X-W, Zhu X-Y, Yan B-J. Design of coal mine water level measuring and monitoring system based on wireless sensor networks. Transducer and Microsystem Technologies. 2008; 12:025.

16. Yi-Bing $\mathrm{Z}$. The long-range monitoring system of water level based on GPRS network. Advances in Control and Communication. Springer. 2012; 137:227-34.

17. Yihuan FMY. Water level monitoring system based on GPRS. Journal of Shenzhen Polytechnic. 2008; 2:009.

18. Zhang L, Ma Y. The monitoring control and PID parameters setting of boiler water-level control system. Machine Tool \& Hydraulics. 2011; 24:040. 